\title{
Orientation of lipids in solid supported lipid bilayers studied by polarized ATR-FTIR spectroscopy ${ }^{1}$
}

\author{
Christian Scheibe and Karin Hauser* \\ Biophysical Chemistry, Department of Chemistry, University of Konstanz, 78457 Konstanz, Germany
}

\begin{abstract}
Solid supported lipid bilayers (SSLB) play an important role as biomimetic membranes to study protein-membrane interactions. We investigated the orientation of lipids in SSLBs at different temperatures and over time. Especially the stability of the lipid bilayer and structural changes upon lipid phase transition were analyzed by polarized ATR-FTIR spectroscopy and with SSLBs of different lipid compositions. The integrity of a lipid bilayer consisting of POPC or a 1:1 mixture of POPC and POPG is conserved over a wide temperature range and over several hours. Furthermore, we were able to monitor changes in the orientation of the lipid alkyl chains upon lipid phase transition for DMPC and DSPC. This study shows that the combination of solid supported lipid bilayers and polarized ATR-FTIR spectroscopy is very powerful to characterize lipid membranes under different environmental conditions. The sensitivity of this technique will be exploited in future studies to analyze the effect of protein-membrane interaction on lipid orientation.
\end{abstract}

Keywords: Solid supported lipid bilayers, polarized ATR-FTIR, lipid phase transition, membrane integrity, molecular orientation

\author{
Abbreviations \\ ATR attenuated total reflection \\ DMPC 1,2-dimyristoyl-sn-glycero-3-phosphocholine \\ DSPC 1,2-distearoyl-sn-glycero-3-phosphocholine \\ FTIR Fourier-transform infrared \\ IRE internal reflection element \\ SSLB solid supported lipid bilayer \\ POPC 1-palmitoyl-2-oleoyl-sn-glycero-3-phosphocholine \\ POPG 1-palmitoyl-2-oleoyl-sn-glycero-3-phospho-rac-(1'-glycerol)
}

\section{Introduction}

Membranes play a crucial role in structure and function of living cells. Many essential processes including signaling or transport take place at cell membranes. Most membranes are phospholipid bilayers

\footnotetext{
${ }^{1}$ Conference proceedings of the 17th European Conference on Spectroscopy of Biological Molecules (ECSBM) Amsterdam, The Netherlands (September 2017).

*Corresponding author. Tel.: +49-(0)7531-88-5356; Fax: +49-7531-88 3139; E-mail: Karin.Hauser@ uni-konstanz.de.
} 


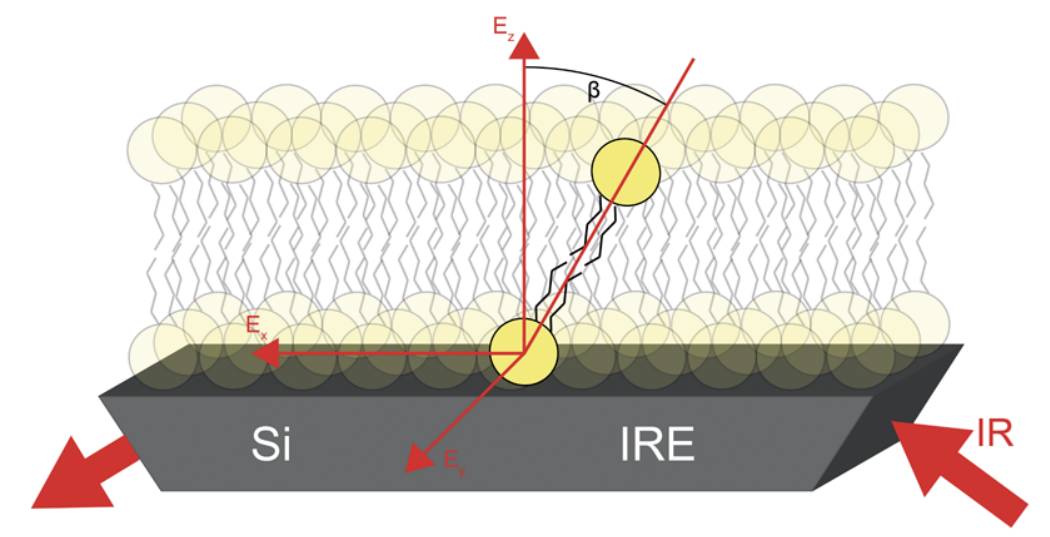

Fig. 1. Schematic representation of the lipid orientation within a SSLB. The determined average angle $\beta$ is illustrated at a highlighted lipid molecule. $E_{x y z}$ are the components of the electric field vector of the evanescent IR wave. $\beta$ represents an average value for all lipids present in the sample.

consisting of different lipids and embedded proteins. In order to investigate the properties of membranes upon their interaction with proteins, solid supported lipid bilayers (SSLB) are widely used as biomimetic membranes [2,11,16,21,23], for example, in aggregation studies of proteins upon membrane interaction $[6,7,13,25]$. The properties of SSLBs can be varied by choosing different lipids. Since SSLBs are formed on a solid surface, one obtains a fixed anisotropic system that, in comparison to studies using liposomes, allows the determination of molecular orientations. This is shown schematically in Fig. 1. In order to determine molecular orientations, it is crucial that this membrane is stable over the course of one experiment. This requires stability over several hours as well as over the temperature range of interest. It is also important to know if the orientation of the lipids changes upon lipid phase transition. Phospholipid membranes have a gel and a liquid phase. The gel phase is found below, and the liquid phase above the phase transition temperature. The liquid phase is described to be a more disordered state than the gel phase [18].

There are numerous techniques used to study lipid membranes like nuclear magnetic resonance (NMR) [12], small angle X-ray scattering (SAXS) [5], atomic force microscopy (AFM) [3] or Fouriertransform infrared (FTIR) spectroscopy [16]. Characteristic vibrational modes of lipids and of proteins can be distinguished. Thus, besides analyzing lipid membranes, FTIR spectroscopy is also well suited to study protein-membrane interaction and to monitor conformational changes of the protein and the membrane simultaneously. By using polarized light, information on the orientation of lipid molecules or other molecular groups present in the sample can be obtained [20]. We studied SSLBs by polarized Attenuated Total Reflection (ATR) FTIR spectroscopy. The internal reflection element (IRE) of the ATR-cell is used as the substrate on which the SSLB is formed. Due to the high sensitivity of this experimental method, even small changes in the orientation of the lipids can be detected. Vibrational modes can also be used to determine the phase transition temperature of lipids, for example the change in band position of the asymmetric $\mathrm{CH}_{2}$ stretching vibration of the lipid alkyl chains. This band shifts to higher wavenumbers upon phase transition from the gel phase into the liquid phase. Thus, the band position yields information about the current phase of the studied lipids and the frequency shift can be used to determine the phase transition temperature [15].

In this study, the focus was set to analyze the stability of SSLBs. Therefore, SSLBs of pure POPC and of a 1:1 mixture of POPC and POPG were used and polarized ATR-FTIR measurements were performed. 
The orientation of the lipid alkyl chains was determined at different temperatures in the range of $15^{\circ} \mathrm{C}-$ $50^{\circ} \mathrm{C}$. Furthermore, the orientation of the lipid alkyl chains was monitored in dependence of time after SSLB formation. Polarized ATIR-FTIR spectra were recorded over $12 \mathrm{~h}$ and at room temperature. In order to analyze the effect of the lipid phase transition, we detected the lipid orientation of DMPC and DSPC below and above the phase transition temperature. Measurements with DMPC were performed in the range of $15^{\circ} \mathrm{C}-50^{\circ} \mathrm{C}$ as well as measurements with DSPC in the range of $35^{\circ} \mathrm{C}-80^{\circ} \mathrm{C}$.

\section{Materials and methods}

Solid supported lipid bilayers were prepared via lipid vesicle spreading on an ATR-crystal $[11,16]$. In order to prepare the vesicles, DMPC (1,2-dimyristoyl-sn-glycero-3-phosphocholine), DSPC (1,2distearoyl-sn-glycero-3-phosphocholine), POPC (1-palmitoyl-2-oleoyl-sn-glycero-3-phosphocholine) and POPG (1-palmitoyl-2-oleoyl-sn-glycero-3-phospho-rac-(1'-glycerol)) were purchased from Avanti Polar Lipids, USA. For storage, the lipids were dissolved in chloroform $(25 \mathrm{mg} / \mathrm{ml}) .200 \mu$ l containing $5 \mathrm{mg}$ of lipids have been taken from the stock solution and were transferred into a glass bottle. Chloroform was removed by drying the sample under a nitrogen stream for 5 minutes. Subsequently, the glass bottle was placed into a vacuum chamber for two hours. Afterwards, the lipids were resuspended in $1 \mathrm{ml}$ Tris- $\mathrm{HCl}(10 \mathrm{mM}, 5 \mathrm{mM} \mathrm{MgCl}, \mathrm{pH}$ 7.4) and incubated at room temperature for 30 minutes. In order to obtain small unilamellar vesicles, the lipid solution was treated with a tip sonificator (Hielscher Ultrasound Technology, Germany) followed by extrusion using a handheld extruder from Avanti Polar Lipids with a $30 \mathrm{~nm}$ polycarbonate membrane. It was important to prepare small unilamellar vesicles because large unilamellar vesicles are supposed to spread incompletely [16].

For bilayer formation, the silicon crystal (IRE) of the ATR-cell was polished using a smooth cloth and polishing paste and incubated with $\mathrm{H}_{2} \mathrm{SO}_{4}(95 \%)$ three times for 10 minutes each. Subsequently, the IRE was rinsed with water and dried under a gentle nitrogen stream after each incubation step. Afterwards, the prepared vesicles were added to the crystal. The formation of the bilayer was monitored spectroscopically over 40 minutes at $25^{\circ} \mathrm{C}$. After this, the IRE was rinsed using buffer to remove vesicles from the supernatant and the surface. Spectra were recorded to validate if the bilayer is still intact.

Measurements were performed using a multi-reflection silicon IRE (Bio-ATR II, Bruker Optics, Germany), a Vertex 70v spectrometer (Bruker Optics, Germany) and an infrared polarizer (PIKE Technologies, USA). The incidence angle was $45^{\circ}$ which results in an efficient penetration depth of $0.47 \mu \mathrm{m}$ for perpendicular polarized light and $0.84 \mu \mathrm{m}$ for parallel polarized light at $1650 \mathrm{~cm}^{-1}$ [9]. The sample volume was $25 \mu 1$. The sample chamber was flushed with dry air during the experiments. For each IR spectrum, 32 scans were recorded with a resolution of $4 \mathrm{~cm}^{-1}$. Fourier-transformation was performed using a Mertz phase correction and Blackman-Harris-3-term apodization. Background measurements were taken for each temperature in parallel and perpendicular polarization with only buffer on the crystal surface.

In order to control the temperature during the experiment, the Bio-ATR II cell was connected to a water bath with an integrated E300 immersion thermostat (LAUDA-Brinkmann, USA). After setting a new temperature, the system was given 10 minutes of equilibration time before new spectra have been recorded.

For the calculation of the average angle, the absorption bands of the symmetric $\left(\sim 2850 \mathrm{~cm}^{-1}\right)$ and asymmetric $\left(\sim 2920 \mathrm{~cm}^{-1}\right)$ stretching vibration of the $\mathrm{CH}_{2}$-group and the stretching vibration of the $\mathrm{C}=\mathrm{O}$ ester bond $\left(\sim 1730 \mathrm{~cm}^{-1}\right)$ were used [20]. The average angle is defined as the angle between the 
lipid alkyl chains and the surface normal. The integrated absorbance is the area under the corresponding absorption band [9] and was determined using Opus 7.5 (Bruker Optics, Germany). Because the lipids are able to rotate around a vector parallel to the lipid alkyl chains, the orientation of the $\mathrm{C}=\mathrm{O}$-bond is expected to be isotropic. The absorbance of the $\mathrm{C}=\mathrm{O}$ stretching vibration was used to determine $R_{\text {iso, }}$, which is a weighting coefficient to correct for dichroitic effects of the spectrometer optics as well as for the different polarization-dependent penetration depths of the IR beam into the sample. $R_{\text {iso }}$ is defined as $R_{\text {iso }}=A_{90} / A_{0}$, where $A_{90}$ and $A_{0}$ are the integrated absorbances determined for parallel $\left(0^{\circ}\right)$ and perpendicular polarized $\left(90^{\circ}\right)$ light respectively [26]. The integrated absorbances of the stretching vibration of the $\mathrm{CH}_{2}$-groups were used to determine the dichroic ratio $R_{\exp }=\left(A_{0} R_{\text {iso }}\right) / A_{90}$. The order parameter $S$ is calculated using $S=\frac{2\left(E_{x}^{2}-R_{\exp } E_{y}^{2}+E_{z}^{2}\right)}{\left(3(\cos \alpha)^{2}-1\right)\left(E_{x}^{2}-R_{\exp } E_{y}^{2}-2 E_{z}^{2}\right)}$ [8], where $E_{x y z}$ are components of the electric field vector of the evanescent wave and $\alpha$ is the angle between the transition dipole moment and the long axis of lipid alkyl chain. The order parameter is then used to calculate the average angle $\beta$ of the long axis of the lipid alkyl chain with respect to the surface normal by using $S=\frac{3(\cos \beta)^{2}-1}{2}[8,9,22]$. The components of the electric field vector and the average angle of the lipid alkyl chains are illustrated in Fig. 1.

In order to determine the phase transition temperature, the data for the average angle at different temperatures were fitted using the sigmoidal function $y=\frac{A_{1}-A_{2}}{1+e^{\left(x-x_{0}\right) / d x}}+A_{2}$. In this fit function, $A_{1}$ corresponds to the average angle below the phase transition temperature, $A_{2}$ to the average angle above the phase transition temperature, $x$ to the temperature, $x_{0}$ to the phase transition temperature, which is determined to be the temperature at the point of inflection and dx corresponds to the slope at the point of inflection. The phase transition temperature was additionally determined from the band positions of the asymmetric stretching vibration. The data were fitted using the same function as above-mentioned. In the latter case, $A_{1}$ and $A_{2}$ correspond to the band positions below and above the phase transition temperature. Fits were performed using the software Origin 2015G (OriginLab, USA).

\section{Results and discussion}

\subsection{Lipid bilayer stability}

In order to investigate thermal stability, solid supported lipid bilayers were prepared and measured at different temperatures $\left(15^{\circ} \mathrm{C}-50^{\circ} \mathrm{C}\right)$. To investigate stability over time, measurements were performed during $12 \mathrm{~h}$ after the formation of the bilayer and at $25^{\circ} \mathrm{C}$. Pure POPC and a 1:1 mixture of POPC and POPG have been used as SSLBs. POPC is often applied to study lipid membranes or membrane-protein interaction and it has been shown before that it forms SSLBs via lipid vesicle spreading [11]. POPC consists of a neutrally charged headgroup and two fatty acid tails. The fatty acid tails contain 18 and 16 carbon atoms with one unsaturated bond in the 18-carbon tail. In comparison, POPG has the same fatty acid tails, but the headgroup is negatively charged. The 1:1 mixture of POPC and POPG was under investigation because charged model membranes are often required to mimic physiological conditions, for example in aggregation studies of alpha-synuclein [7,19].

The results of the measurements at different temperatures are presented in Fig. 2a. The orientation of the lipids with respect to the surface normal shows basically no changes over the whole temperature range. The determined angles (average values over the whole temperature range) are $\beta=38.1^{\circ}$ for POPC and $\beta=38.2^{\circ}$ for POPC:POPG. Therefore, there is no significant difference in the average angle between a membrane of pure POPC and a 1:1 mixture of POPC and POPG. Both lipids are expected to 

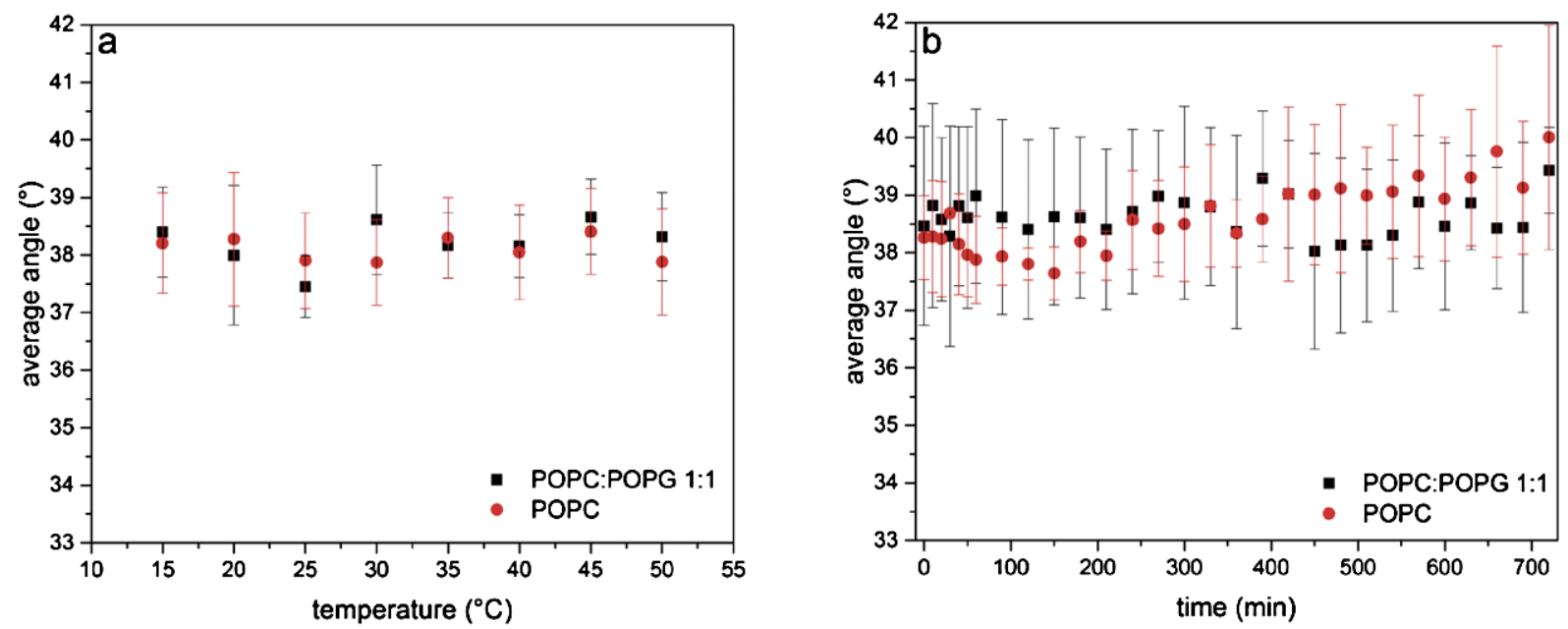

Fig. 2. Stability of SSLBs at different temperatures and over time. The orientation of the lipid alkyl chains with respect to the surface normal of a mixture of POPC and POPG (black squares) and of pure POPC (red circles) is shown (a) for different temperatures and (b) over $12 \mathrm{~h}$ at $25^{\circ} \mathrm{C}$.

be well above their phase transition temperature and in their liquid phase. The phase transition occurs at $-2^{\circ} \mathrm{C}$ [24] for POPC and close to or below $0^{\circ} \mathrm{C}$ for POPG [17]. Furthermore, we could prove with our measurements that the orientation of the SSLB stays constant over a wide temperature range as long as it stays in the liquid lipid phase. This is of high relevance when it comes to the investigation of protein-membrane interaction at different temperatures.

The same bilayers were used to study their stability over $12 \mathrm{~h}$. As shown in Fig. $2 \mathrm{~b}$, there is no significant change in the orientation of the lipid alkyl chains over this long period of time for both membranes. Several measurements were performed and averaged. The average angle was determined to be $38.6^{\circ}$ for pure POPC and $39.2^{\circ}$ for the $1: 1$ mixture of POPC and POPG. The stability is crucial for long-lasting experiments using SSLBs as biomimetic membranes.

\subsection{Effect of lipid phase transition on orientation of lipids}

Since we were able to show that the orientation of lipids in POPC and POPC:POPG SSLBs does not change as long as the temperature stays above the phase transition temperature, we wanted to investigate the effect of the phase transition. For that, we used DMPC and DSPC because their phase transition temperatures lie in an experimentally accessible temperature range. DMPC consists of a neutrally charged headgroup and two fully saturated fatty acid tails containing 14 carbon atoms. DSPC consists of the same headgroup but the fatty acid tails contain 18 carbon atoms. The fatty acid tails of DSPC are also fully saturated. The corresponding phase transition temperatures are $24^{\circ} \mathrm{C}$ for DMPC [14] and $55^{\circ} \mathrm{C}$ for DSPC [10].

The average angle of the lipid alkyl chains of DMPC and DSPC with respect to the surface normal was calculated for different temperatures. In Fig. 3a the results of these measurements are shown. Two phases become visible, above and below the phase transition temperatures as well as the transition in between. The phase transition temperatures were determined to be $26.1^{\circ} \mathrm{C}$ for DMPC and $57.5^{\circ} \mathrm{C}$ for DSPC.

Upon phase transition, the orientation of the lipids in the DMPC bilayer changes by $\Delta^{\circ}=2.5^{\circ}$ and the orientation of the lipids in the DSPC bilayer by $\Delta^{\circ}=3.4^{\circ}$. For DMPC the average angle was $36.6^{\circ} \pm$ 

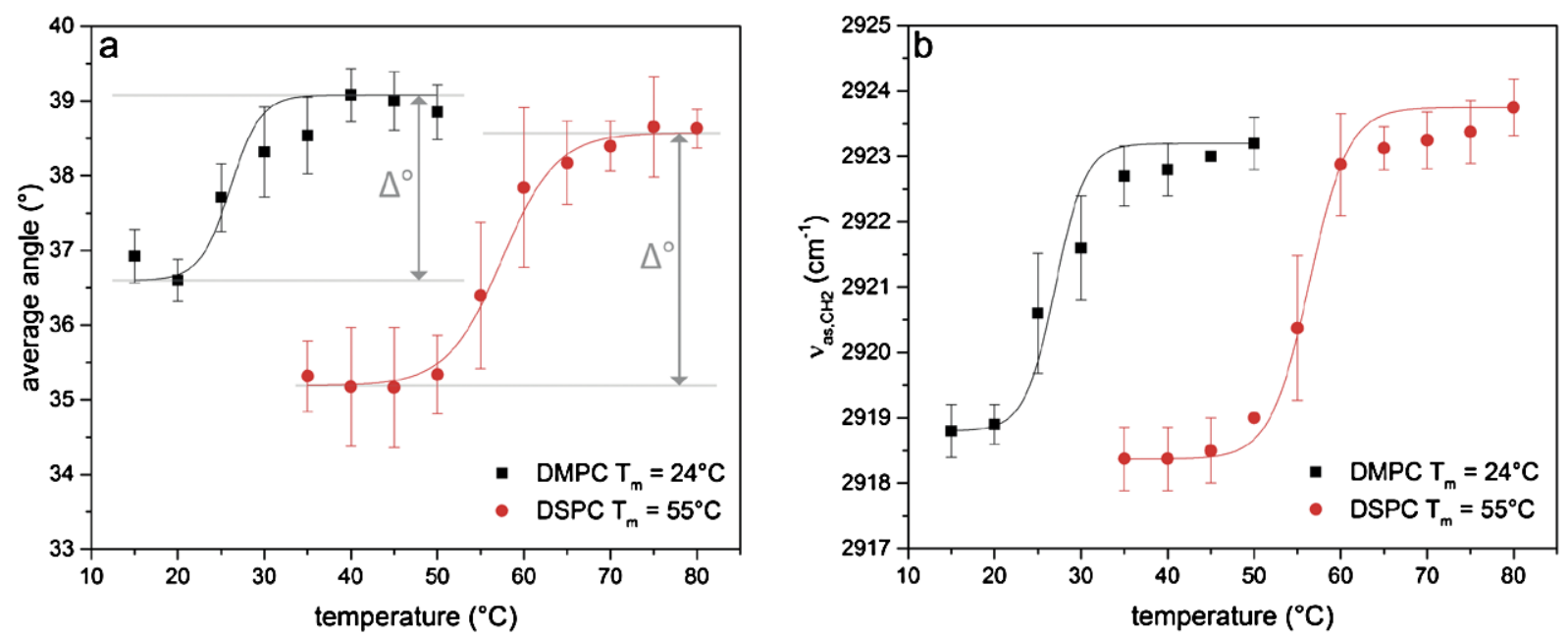

Fig. 3. Changes upon lipid phase transition of DMPC and DSPC. Investigation of (a) the orientation of the lipid alkyl chain and (b) the frequency position of the band corresponding to the asymmetric stretching vibration of the $\mathrm{CH}_{2}$-groups $\left(v_{\mathrm{as}}, \mathrm{CH} 2\right)$ in the lipid alkyl chain for DMPC (black squares) and DSPC (red circles) respectively. The data were fitted to determine the phase transition temperature and the difference in the average angle for both phases $\left(\Delta^{\circ}\right)$.

$0.3^{\circ}$ below the phase transition temperature and $39.1^{\circ} \pm 0.4^{\circ}$ above the phase transition temperature. For DSPC the corresponding average angles were at $35.2^{\circ} \pm 0.3^{\circ}$ and $38.6^{\circ} \pm 0.3^{\circ}$. The errors were determined as the standard deviation of the average angles above and below the phase transition temperature and are an order of magnitude smaller than the angle change $\Delta^{\circ}$. This means that the here detected small changes of about $3^{\circ}$ upon phase transition are significant because we were able to determine the angles very precisely.

Our results show that the lipids are oriented slightly closer to the surface normal in the gel phase than in the liquid phase. The liquid phase is described to be more disordered than the gel phase [18]. In a more disordered membrane, it can be assumed that the lipids can move more freely and thus are able to tilt away from the surface normal. Our technique is sensitive enough to detect and quantify this tilt.

It is striking that the errors for DMPC and DSPC are smaller than the errors for POPC and POPC:POPG. In comparison to DMPC and DSPC, POPC and POPG contain unsaturated bonds. This results in a less tightly packing in the bilayer and a higher mobility of the single lipid molecules. A higher mobility could cause a higher variance around the average angle compared to the DMPC and DSPC lipids with fully saturated alkyl chains.

In Fig. $3 \mathrm{~b}$ the position of the band corresponding to the asymmetric stretching vibration of the $\mathrm{CH}_{2}$ groups is shown. These data were extracted from the same spectra used for the calculation of the average angles in Fig. 3a. The phase transition temperatures were determined to be $27.0^{\circ} \mathrm{C}$ for DMPC and $56.4^{\circ} \mathrm{C}$ for DSPC.

The transition temperatures derived from our experiments are slightly higher than the literature values given above. A reason for this could be a non-homogeneous heat transfer from the surrounding casing of the Bio-ATR II cell to the sample.

We analyzed the phase transition temperatures by the average angle of the lipid alkyl chains with respect to the surface normal and by the position of the band corresponding to the asymmetric stretching vibration of the $\mathrm{CH}_{2}$ group. The results for both of these methods are in good agreement with each other. Thus, we were able to show that the phase transition has an effect on the orientation of the lipids inside 
a lipid membrane. This agrees with recent studies that reported a molecular tilt at the phase transition temperature using synchrotron diffuse x-ray scattering [14]. With polarized ATR-FTIR measurements, it is furthermore possible to quantify the angle by which the lipid alkyl chains are tilting.

Knowledge about the effect of the phase transition on the orientation of lipids in SSLBs might be useful for comparison to changes induced by protein interaction since it has been observed that at least membrane proteins can alter the phase transition of lipid membranes [1,4]. It also proves the sensitivity of this technique to detect even small changes in the orientation of the lipids.

\section{Conclusions}

Polarized ATR-FTIR spectroscopy proves to be a powerful tool to investigate the orientation of the lipids inside SSLBs. We were able to show that POPC and POPG:POPC SSLBs are stable at different temperatures and over several hours. As long as no phase transition occurs, the orientation of the lipids remains unaffected by changes in the temperature. This is of high relevance for protein-membrane studies that are using SSLBs as biomimetic membranes.

Furthermore, we were able to show that the lipid phase transition is reflected in the orientation of the lipids inside a lipid membrane and we could quantify this small effect for different lipids. Polarized ATR-FTIR is highly sensitive to detect differences of only a few degrees in the lipid orientation. Since it has been reported that membrane proteins can alter the phase transition of the surrounding membrane, it would be interesting to analyze if protein-membrane interaction can have a similar effect. This might be an implication if the protein stays at the interphase or inserts into the membrane.

\section{Acknowledgements}

This work was supported by the Deutsche Forschungsgemeinschaft (SFB 969, A2) and the Konstanz Research School Chemical Biology. We gratefully acknowledge fruitful discussions with Tim Keiderling.

\section{References}

[1] J.L.R. Arrondo and F.M. Goñi, Infrared studies of protein-induced perturbation of lipids in lipoproteins and membranes, Chemistry and Physics of Lipids 96(1-2) (1998), 53-68. doi:10.1016/S0009-3084(98)00080-2.

[2] C. Bolterauer and H. Heller, Calculation of IR dichroic values and order parameters from molecular dynamics simulations and their application to structure determination of lipid bilayers, European Biophysics Journal with Biophysics Letters 24(5) (1996), 322-334. doi:10.1007/BF00180373.

[3] M. Chadli et al., New tethered phospholipid bilayers integrating functional G-protein-coupled receptor membrane proteins, Langmuir 33(39) (2017), 10385-10401. doi:10.1021/acs.langmuir.7b01636.

[4] M. Cortijo et al., Intrinsic protein-lipid interactions: Infrared spectroscopic studies of gramicidin A, bacteriorhodopsin and $\mathrm{Ca}^{2+}$-ATPase in biomembranes and reconstituted systems, Journal of Molecular Biology 157(4) (1982), 597-618. doi:10.1016/0022-2836(82)90501-0.

[5] E. Di Cola et al., Lipid-stabilized water-oil interfaces studied by microfocusing small-angle X-ray scattering, Langmuir 33(36) (2017), 9100-9105. doi:10.1021/acs.langmuir.7b02076.

[6] Y.A. Domanov and P.K.J. Kinnunen, Islet amyloid polypeptide forms rigid lipid-protein amyloid fibrils on supported phospholipid bilayers, Journal of Molecular Biology 376(1) (2008), 42-54. doi:10.1016/j.jmb.2007.11.077.

[7] M.A. Fallah et al., Simultaneous IR-spectroscopic observation of alpha-synuclein, lipids, and solvent reveals an alternative membrane-induced oligomerization pathway, Chembiochem (2017). doi:10.1002/cbic.201700355. 
[8] U.P. Fringeli, In situ infrared attenuated total reflection (IR ATR) spectroscopy: A complementary analytical tool for drug design and drug delivery, CHIMIA International Journal for Chemistry 46(5) (1992), 200-214.

[9] E. Goormaghtigh, V. Raussens and J.M. Ruysschaert, Attenuated total reflection infrared spectroscopy of proteins and lipids in biological membranes, Biochim Biophys Acta 1422(2) (1999), 105-185. doi:10.1016/S0304-4157(99)00004-0.

[10] S. Kim and R. Chang, Structure, dynamics, and phase behavior of DOPC/DSPC mixture membrane systems: Molecular dynamics simulation studies, Bulletin of the Korean Chemical Society 37(7) (2016), 1076-1085. doi:10.1002/bkcs.10827.

[11] C. Kötting, J. Güldenhaupt and K. Gerwert, Time-resolved FTIR spectroscopy for monitoring protein dynamics exemplified by functional studies of Ras protein bound to a lipid bilayer, Chemical Physics 396(Supplement C) (2012), 72-83.

[12] T.R. Molugu, S. Lee and M.F. Brown, Concepts and methods of solid-state NMR spectroscopy applied to biomembranes, Chemical Reviews 117(19) (2017), 12087-12132. doi:10.1021/acs.chemrev.6b00619.

[13] H. Mueller, H.-J. Butt and E. Bamberg, Adsorption of membrane-associated proteins to lipid bilayers studied with an atomic force microscope: Myelin basic protein and cytochrome c, The Journal of Physical Chemistry B 104(18) (2000), 4552-4559. doi:10.1021/jp9940856.

[14] J.F. Nagle, X-ray scattering reveals molecular tilt is an order parameter for the main phase transition in a model biomembrane, Physical Review E 96(3) (2017), 030401. doi:10.1103/PhysRevE.96.030401.

[15] S. Olsztyliska-Janus et al., ATR-IR study of skin components: Lipids, proteins and water. Part I: Temperature effect, Spectrochimica Acta Part A - Molecular and Biomolecular Spectroscopy 188 (2018), 37-49.

[16] P. Pinkerneil et al., Surface-attached polyhistidine-tag proteins characterized by FTIR difference spectroscopy, Chemphyschem 13(11) (2012), 2649-2653. doi:10.1002/cphc.201200358.

[17] B. Pozo Navas et al., Composition dependence of vesicle morphology and mixing properties in a bacterial model membrane system, Biochimica et Biophysica Acta (BBA) - Biomembranes 1716(1) (2005), 40-48. doi:10.1016/j.bbamem. 2005.08.003.

[18] K.A. Riske et al., Lipid bilayer pre-transition as the beginning of the melting process, Biochimica et Biophysica Acta (BBA) - Biomembranes 1788(5) (2009), 954-963. doi:10.1016/j.bbamem.2009.01.007.

[19] M. Robotta et al., Alpha-synuclein binds to the inner membrane of mitochondria in an $\alpha$-helical conformation, ChemBioChem 15(17) (2014), 2499-2502. doi:10.1002/cbic.201402281.

[20] L.K. Tamm and S.A. Tatulian, Infrared spectroscopy of proteins and peptides in lipid bilayers, Quarterly Reviews of Biophysics 30(4) (1997), 365-429. doi:10.1017/S0033583597003375.

[21] S.A. Tatulian, Attenuated total reflection Fourier transform infrared spectroscopy: A method of choice for studying membrane proteins and lipids, Biochemistry 42(41) (2003), 11898-11907. doi:10.1021/bi034235+.

[22] S.A. Tatulian et al., Secondary structure and orientation of phospholamban reconstituted in supported bilayers from polarized attenuated total reflection FTIR spectroscopy, Biochemistry 34(13) (1995), 4448-4456. doi:10.1021/bi00013a038.

[23] M.L. Wagner and L.K. Tamm, Tethered polymer-supported planar lipid bilayers for reconstitution of integral membrane proteins: Silane-polyethyleneglycol-lipid as a cushion and covalent linker, Biophysical Journal 79(3) (2000), 1400-1414. doi:10.1016/S0006-3495(00)76392-2.

[24] U. Wanderlingh et al., Molecular dynamics of POPC phospholipid bilayers through the gel to fluid phase transition: An incoherent quasi-elastic neutron scattering study, Journal of Chemistry 2017 (2017), 3654237.

[25] E.A. Yates et al., Specific domains of A $\beta$ facilitate aggregation on and association with lipid bilayers, Journal of Molecular Biology 425(11) (2013), 1915-1933. doi:10.1016/j.jmb.2013.03.022.

[26] G. Zhang and T.A. Keiderling, Equilibrium and dynamic spectroscopic studies of the interaction of monomeric $\beta$ lactoglobulin with lipid vesicles at low pH, Biochemistry 53(19) (2014), 3079-3087. doi:10.1021/bi500027x. 